\title{
Parameter estimation for fractional Ornstein-Uhlenbeck processes with discrete observations
}

\author{
Yaozhong $\mathrm{HU}^{*}$ and Jian Song
}

\begin{abstract}
Consider an Ornstein-Uhlenbeck process, $d X_{t}=-\theta X_{t} d t+\sigma d B_{t}^{H}$, driven by fractional Brownian motion $B^{H}$ with known Hurst parameter $H \geq \frac{1}{2}$ and known variance $\sigma$. But the parameter $\theta>0$ is unknown. Assume that the process is observed at discrete time instants $t=h, 2 h, \cdots, n h$. We construct an estimator $\hat{\theta}_{n}$ of $\theta$ which is strongly consistent, namely, $\hat{\theta}_{n}$ converges to $\theta$ almost surely as $n \rightarrow \infty$. We also obtain a central limit type theorem and a Berry-Esseen type theorem for this estimator $\hat{\theta}_{n}$ when $1 / 2 \leq H<3 / 4$. The tool we use is some recent results on central limit theorems for multiple Wiener integrals through Malliavin calculus. It should be pointed out that no condition on the step size $h$ is required, contrary to the existing conventional assumptions.
\end{abstract}

\section{Introduction}

The Ornstein-Uhlenbeck process $X_{t}$ driven by a certain type of noise $Z_{t}$ is described by the following Langevin equation

$$
d X_{t}=-\theta X_{t} d t+\sigma d Z_{t} .
$$

If the parameter $\theta$ is unknown and if the process $\left(X_{t}, 0 \leq t \leq T\right)$ can be observed continuously, then an important problem is to estimate the parameter $\theta$ based on the (single path) observation $\left(X_{t}, 0 \leq t \leq T\right)$. See [7] and the references therein for a short account of the research works relevant to this problem. In this paper, we consider the case $Z_{t}$ is a fractional Brownian motion with Hurst parameter $H$. Namely, we consider the following stochastic Langevin equation

$$
d X_{t}=-\theta X_{t} d t+\sigma d B_{t}^{H}, \quad X_{0}=x,
$$

where $\theta$ is an unknown parameter. We assume $\theta>0$ through out the paper so that the process is ergodic (when $\theta<0$ the solution to (1.2) will diverge). If

\footnotetext{
${ }^{*}$ Y. Hu is partially supported by a grant from the Simons Foundation \#209206.
} 
the process $\left(X_{t}, 0 \leq t \leq T\right)$ can be observed continuously, then the least square estimator $\tilde{\theta}_{T}$, defined by

$$
\tilde{\theta}_{T}=-\frac{\int_{0}^{T} X_{t} d X_{t}}{\int_{0}^{T} X_{t}^{2} d t}
$$

was studied in [9], where it is proved that $\tilde{\theta}_{T} \rightarrow \theta$ almost surely as $T \rightarrow \infty$ and that $\sqrt{T}\left(\tilde{\theta}_{T}-\theta\right)$ converges in law to a mean zero normal random variable. The variance of this normal is also calculated. As a consequence it is also proved in [9] that the following estimator

$$
\bar{\theta}_{T}:=\left(\frac{1}{\sigma^{2} H \Gamma(2 H) T} \int_{0}^{T} X_{t}^{2} d t\right)^{-\frac{1}{2 H}}
$$

is also strongly consistent and $\sqrt{T}\left(\bar{\theta}_{T}-\theta\right)$ converges in law to a mean zero normal with explicit variance given by $\frac{\theta \sigma_{H}^{2}}{(2 H)^{2}}$.

In applications usually the process cannot be observed continuously. Only discrete time observations are available. To simplify presentation of the paper, we assume that the process $X_{t}$ is observed at discrete time instants $t_{k}=k h, k=1,2, \cdots, n$, for some fixed $h>0$. We seek to estimate $\theta$ based on $X_{h}, X_{2 h}, \cdots, X_{n h}$.

Motivated by the estimator (1.4), we propose to use a function of $\frac{1}{n} \sum_{k=1}^{n}\left|X_{k h}\right|^{p}$ as a statistic to estimate $\theta$. More precisely, we define

$$
\hat{\theta}_{n}=\left(\frac{1}{n \sigma^{2} H \Gamma(2 H)} \sum_{k=1}^{n} X_{k h}^{2}\right)^{-\frac{1}{2 H}} .
$$

We shall show that $\hat{\theta}_{n}$ converges to $\theta$ almost surely as $n$ tends to $\infty$. We shall also show that $\sqrt{n}\left(\hat{\theta}_{n}-\theta\right)$ converges in law to mean zero normal random variable with variance $\frac{\theta^{2}}{2 H^{2}}$ as $n \rightarrow \infty$. The following Berry-Esseen type theorem will also be shown

$$
\sup _{z \in \mathbb{R}}\left|P\left(\sqrt{\frac{2 H^{2} n}{\theta^{2}}}\left(\hat{\theta}_{n}-\theta\right) \leq z\right)-\Psi(z)\right| \leq C n^{4 H-3},
$$

where $\Psi(z)=\frac{1}{\sqrt{2 \pi}} \int_{-\infty}^{z} e^{-\frac{u^{2}}{2}} d u$ is the error function.

Usually, to obtain consistency result for discrete time observations, one has to assume that the length $h$ of the time interval between two consecutive observations depends on $n$ (namely $h=h_{n}$ ) and $h_{n}$ converges to 0 as $n \rightarrow \infty$ and $h_{n}$ and $n$ must satisfy some other conditions (see [3], [15], [7], [8], and references therein). Surprisingly enough, for our simple model (1.2) and for our estimator defined above we don't need to assume $h$ depends on $n$. In fact, we don't have any condition on $h$ ! Let us also point out that throughout the paper, we 
assume that the observation times are uniform: $t_{k}=k h, k=1, \cdots, n$. General deterministic observation times $t_{k}$ can be also considered in a similar way.

The paper is organized as follows. In Section 2, some known results that we will use are recalled. The strong consistency of a slight more general estimator is proved in Section 3. Section 4 deals with the central limit type theorem and Section 5 concerns with the Berry-Esseen type theorem.

Along the paper, we denote by $C$ a generic constant possibly depending on $\theta$ and/or $h$ which may be different from line to line.

\section{Preliminaries}

In this section we first introduce some basic facts on the Malliavin calculus for the fractional Brownian motion and recall the main results in [12] and [14] concerning the central limit theorem and Berry-Essen type results for multiple stochastic integrals.

We are working on some complete probability space $(\Omega, \mathcal{F}, P)$. The expectation on this probability space is denoted by $\mathbb{E}$. The fractional Brownian motion with Hurst parameter $H \in(0,1),\left(B_{t}^{H}, t \in \mathbb{R}\right)$ is a zero mean Gaussian process with the following covariance structure:

$$
\mathbb{E}\left(B_{t}^{H} B_{s}^{H}\right)=R_{H}(t, s)=\frac{1}{2}\left(|t|^{2 H}+|s|^{2 H}-|t-s|^{2 H}\right) .
$$

Fix a time interval $[0, T]$. Denote by $\mathcal{E}$ the set of real valued step functions on $[0, T]$ and let $\mathcal{H}$ be the Hilbert space defined as the closure of $\mathcal{E}$ with respect to the inner product

$$
\left\langle\mathbf{1}_{[0, t]}, \mathbf{1}_{[0, s]}\right\rangle_{\mathcal{H}}=R_{H}(t, s),
$$

where $R_{H}$ is the covariance function of the $\mathrm{fBm}$, given in (2.1). The mapping $\mathbf{1}_{[0, t]} \longmapsto B_{t}^{H}$ can be extended to a linear isometry between $\mathcal{H}$ and the Gaussian space $\mathcal{H}_{1}$ spanned by $B^{H}$ (see also [11]). We denote this isometry by $\varphi \longmapsto$

$B^{H}(\varphi)$, which can be also considered as the stochastic integral of $\varphi$ with respect to $B^{H}$ (denoted by $\left.B^{H}(\varphi)=\int_{0}^{T} \varphi(t) d B_{t}^{H}\right)$. For $H=\frac{1}{2}$ we have $\mathcal{H}=L^{2}([0, T])$, whereas for $H>\frac{1}{2}$ we have $L^{\frac{1}{H}}([0, T]) \subset \mathcal{H}$ and for $\varphi, \psi \in L^{\frac{1}{H}}([0, T])$ we have

$$
\begin{aligned}
\mathbb{E}\left(B^{H}(\varphi) B^{H}(\psi)\right) & =\mathbb{E}\left(\int_{0}^{T} \varphi(t) d B_{t}^{H} \int_{0}^{T} \psi(t) d B_{t}^{H}\right) \\
& =\langle\varphi, \psi\rangle_{\mathcal{H}}=\int_{0}^{T} \int_{0}^{T} \varphi_{s} \psi_{t} \phi(t-s) d s d t
\end{aligned}
$$

where

$$
\phi(u)=H(2 H-1)|u|^{2 H-2} .
$$

Let $\mathcal{S}$ be the space of smooth and cylindrical random variables of the form

$$
F=f\left(B^{H}\left(\varphi_{1}\right), \ldots, B^{H}\left(\varphi_{n}\right)\right), \quad \varphi_{1}, \cdots, \varphi_{n} \in L^{\frac{1}{H}}([0, T]) \subseteq \mathcal{H},
$$


where $f \in C_{b}^{\infty}\left(\mathbb{R}^{n}\right)$ ( $f$ and all its partial derivatives are bounded). For a random variable $F$ of the form (2.4) we define its Malliavin derivative as the $\mathcal{H}$-valued random element

$$
D F=\sum_{i=1}^{n} \frac{\partial f}{\partial x_{i}}\left(B^{H}\left(\varphi_{1}\right), \ldots, B^{H}\left(\varphi_{n}\right)\right) \varphi_{i}
$$

By iteration, one can define the $m$-th derivative $D^{m} F$, which is an element of $L^{2}\left(\Omega ; \mathcal{H}^{\otimes m}\right)$, for every $m \geq 2$. For $m \geq 1, \mathbb{D}^{m, 2}$ denotes the closure of $\mathcal{S}$ with respect to the norm $\|\cdot\|_{m, 2}$, defined by the relation

$$
\|F\|_{m, 2}^{2}=\mathbb{E}\left[|F|^{2}\right]+\sum_{i=1}^{m} \mathbb{E}\left(\left\|D^{i} F\right\|_{\mathcal{H}^{\otimes i}}^{2}\right) .
$$

Let $\delta$ be the adjoint of the operator $D$, also called the divergence operator. A random element $u \in L^{2}(\Omega, \mathcal{H})$ belongs to the domain of $\delta$, denoted by $\operatorname{Dom}(\delta)$, if and only if it verifies

$$
\left|\mathbb{E}\langle D F, u\rangle_{\mathcal{H}}\right| \leq c_{u}\|F\|_{L^{2}},
$$

for any $F \in \mathbb{D}^{1,2}$, where $c_{u}$ is a constant depending only on $u$. If $u \in \operatorname{Dom}(\delta)$, then the random variable $\delta(u)$ is defined by the duality relationship

$$
\mathbb{E}(F \delta(u))=\mathbb{E}\langle D F, u\rangle_{\mathcal{H}},
$$

which holds for every $F \in \mathbb{D}^{1,2}$. The divergence operator $\delta$ is also called the Skorohod integral because in the case of the Brownian motion it coincides with the anticipating stochastic integral introduced by Skorohod in [16]. We will make use of the notation $\delta(u)=\int_{0}^{T} u_{t} d B_{t}^{H}$.

For every $n \geq 1$, let $\mathcal{H}_{n}$ be the $n$th Wiener chaos of $B^{H}$, that is, the closed linear subspace of $L^{2}(\Omega, \mathcal{F}, P)$ generated by the random variables: $\left\{H_{n}\left(B^{H}(h)\right), h \in\right.$ $\left.\mathcal{H},\|h\|_{\mathcal{H}}=1\right\}$, where $H_{n}$ is the $n$th Hermite polynomial. The mapping $h^{\otimes n} \in$ $\mathcal{H}^{\odot n} \rightarrow I_{n}\left(h^{\otimes n}\right) \in \mathcal{H}_{n}$, defined by $I_{n}\left(h^{\otimes n}\right)=H_{n}\left(B^{H}(h)\right)$ provides a linear isometry between the symmetric tensor product $\mathcal{H}^{\odot n}$ and $\mathcal{H}_{n}$. For $H=\frac{1}{2}, I_{n}$ coincides with the multiple Itô stochastic integral. On the other hand, $I_{n}\left(h^{\otimes n}\right)$ coincides with the iterated divergence $\delta^{n}\left(h^{\otimes n}\right)$ and coincides with the multiple Itô type stochastic integral introduced in [4].

We will make use of the following central limit theorem for multiple stochastic integrals (see [14]).

Proposition 2.1 Let $\left\{F_{n}, n \geq 1\right\}$ be a sequence of random variables in the $p$-th Wiener chaos, $p \geq 2$, such that $\lim _{n \rightarrow \infty} \mathbb{E}\left(F_{n}^{2}\right)=\sigma^{2}$. Then, the following conditions are equivalent:

(i) $F_{n}$ converges in law to $N\left(0, \sigma^{2}\right)$ as $n$ tends to infinity.

(ii) $\left\|D F_{n}\right\|_{\mathcal{H}}^{2}$ converges in $L^{2}$ to a constant as $n$ tends to infinity. 
Remark 2.2 In [14] it is proved that (i) is equivalent to the fact that $\left\|D F_{n}\right\|_{\mathcal{H}}^{2}$ converges in $L^{2}$ to $p \sigma^{2}$ as $n$ tends to infinity. If we assume (ii), the limit of $\left\|D F_{n}\right\|_{\mathcal{H}}^{2}$ must be equal to $p \sigma^{2}$ because

$$
\mathbb{E}\left(\left\|D F_{n}\right\|_{\mathcal{H}}^{2}\right)=p \mathbb{E}\left(F_{n}^{2}\right) .
$$

To obtain Berry-Esseen type estimate, we shall use a result from [12], which we shall state in our fractional Brownian motion framework. The validity is straightforward.

Assume that $F=\int_{0}^{T} \int_{0}^{T} f(s, t) d B_{s}^{H} d B_{t}^{H}$ is an element in the second chaos, where $f$ is symmetric functions of two variables. Then with this kernel $f$ we can define a Hilbert-Schmidt operator $H_{f}$ from $\mathcal{H}$ to $\mathcal{H}$ by

$$
H_{f} g(t)=\langle f(t, \cdot), g(\cdot)\rangle_{\mathcal{H}} .
$$

If $g$ is a continuous function on $[0, T]$, then

$$
H_{f} g(t)=\int_{0}^{T} \int_{0}^{T} f(t, u) g(v) \phi(u-v) d u d v
$$

where $\phi$ is defined by (2.3). For $p \geq 2$, the $p$-th cumulant of $F$ is well known (see, e.g. [5] for a proof).

$$
\begin{aligned}
\kappa_{p}(F)= & 2^{p-1}(p-1) ! \operatorname{Tr}\left(H_{f}^{p}\right) \\
= & 2^{p-1}(p-1) ! \int_{[0, T]^{2 p}} f\left(s_{1}, s_{2}\right) f\left(s_{3}, s_{4}\right) \cdots f\left(s_{2 p-1}, s_{2 p}\right) \phi\left(s_{2}, s_{3}\right) \cdots \\
& \phi\left(s_{2 p-2}, s_{2 p-1}\right) \phi\left(s_{2 p}, s_{1}\right) d s_{1} \cdots d s_{2 p} .
\end{aligned}
$$

Let

$$
F_{n}=I_{2}\left(f_{n}\right)=\int_{0}^{T} \int_{0}^{T} f_{n}(s, t) d B_{s}^{H} d B_{t}^{H}
$$

be a sequence of random variables in the second chaos. We shall use the following result from [12], Proposition 3.8.

Proposition 2.3 If $\kappa_{2}\left(F_{n}\right)=\mathbb{E}\left(F_{n}^{2}\right) \rightarrow 1$ and $\kappa_{4}\left(F_{n}\right) \rightarrow 0$, then

$$
\sup _{z \in \mathbb{R}}\left|P\left(F_{n} \leq z\right)-\Psi(z)\right| \leq \sqrt{\frac{\kappa_{4}\left(F_{n}\right)}{6}+\left(\kappa_{2}\left(F_{n}\right)-1\right)^{2}},
$$

where $\Psi(z)=\frac{1}{\sqrt{2 \pi}} \int_{-\infty}^{z} e^{-\frac{u^{2}}{2}} d u$ is the error function.

\section{Construction and strong consistency of the es- timator}

As in [9], we can assume that $X_{0}=0$, and

$$
X_{t}=\sigma \int_{0}^{t} e^{-\theta(t-s)} d B_{s}^{H} .
$$


[We can express $X_{t}=Y_{t}-e^{-\theta t} \xi$, where $Y_{t}=\sigma \int_{-\infty}^{t} e^{-\theta(t-s)} d B_{s}^{H}$ is stationary and $\xi=\sigma \int_{-\infty}^{0} e^{\theta s} d B_{s}^{H}$ has the limiting (normal) distribution of $X_{t}$.]

Let $p>0$ be a positive number and denote

$$
\eta_{p, n}=\frac{1}{n} \sum_{k=1}^{n}\left|X_{k h}\right|^{p}
$$

It is easy to argue that

$$
\lim _{n \rightarrow \infty} \eta_{p, n}=\lim _{n \rightarrow \infty} \frac{1}{n} \sum_{k=1}^{n}\left|Y_{k h}\right|^{p}
$$

Thus by the ergodic theorem we see that $\eta_{p, n}$ converges almost surely to

$$
\begin{aligned}
\lim _{n \rightarrow \infty} \eta_{p, n} & =\mathbb{E}\left(\left|Y_{h}\right|^{p}\right)=\lim _{n \rightarrow \infty} \mathbb{E}\left(\left|X_{n h}\right|^{p}\right) \\
& =c_{p} \lim _{n \rightarrow \infty}\left(\operatorname{Var}\left(X_{n h}\right)\right)^{p / 2} \\
& =c_{p} \sigma^{p} \theta^{-H p}(H \Gamma(2 H))^{p / 2},
\end{aligned}
$$

where

$$
c_{p}=\frac{1}{\sqrt{2 \pi} \sigma} \int_{-\infty}^{\infty}|x|^{p} e^{-\frac{x^{2}}{2 \sigma^{2}}} d x=\pi^{-1 / 2} \Gamma\left(\frac{p+1}{2}\right) .
$$

Thus we obtain

Proposition 3.1 Let $p>0$, and $h>0$. Define

$$
\begin{aligned}
\hat{\theta}_{p, n} & =\left(\frac{1}{c_{p} \sigma^{p}(H \Gamma(2 H))^{p / 2}} \eta_{p, n}\right)^{-\frac{1}{p H}} \\
& =\left(\frac{1}{n \pi^{-1 / 2} \Gamma\left(\frac{p+1}{2}\right) \sigma^{p}(H \Gamma(2 H))^{p / 2}} \sum_{k=1}^{n}\left|X_{k h}\right|^{p}\right)^{-\frac{1}{p H}}
\end{aligned}
$$

Then $\hat{\theta}_{p, n} \rightarrow \theta$ almost surely as $n \rightarrow \infty$.

\section{Central limit theorem}

In this section we shall show that $\sqrt{n}\left(\hat{\theta}_{p, n}-\theta\right)$ converges in law to a mean zero normal and we shall also compute the limiting variance. But we shall study the case $p=2$. More general case may be discussed with the same approach, but it will be much more sophisticated. When $p=2$, we denote $\hat{\theta}_{n}=\hat{\theta}_{2, n}$. Namely,

$$
\hat{\theta}_{n}=\left(\frac{1}{n \sigma^{2} H \Gamma(2 H)} \sum_{k=1}^{n} X_{k h}^{2}\right)^{-\frac{1}{2 H}} .
$$


Denote

$$
\xi_{n}=\frac{1}{n} \sum_{k=1}^{n} X_{k h}^{2}
$$

and $\rho=\sigma^{2} H \Gamma(2 H)$. Then $\hat{\theta}_{n}=\left(\frac{\xi_{n}}{\rho}\right)^{-\frac{1}{2 H}}$. From the last section, we see

$$
\lim _{n \rightarrow \infty} \xi_{n}=\lim _{n \rightarrow \infty} \mathbb{E}\left(\xi_{n}\right)=\sigma^{2} \theta^{-2 H} H \Gamma(2 H)=\rho \theta^{-2 H} .
$$

First we want to show that

$$
F_{n}:=\sqrt{n}\left(\xi_{n}-\mathbb{E}\left(\xi_{n}\right)\right)
$$

converges in law. We shall use Proposition 2.1.

Lemma 4.1 When $H \in\left[\frac{1}{2}, \frac{3}{4}\right)$, we have,

$$
\lim _{n \rightarrow \infty} \mathbb{E}\left(F_{n}^{2}\right)=2 \rho^{2} \theta^{-4 H}
$$

and

$$
\left|\mathbb{E}\left(F_{n}^{2}\right)-2 \rho^{2} \theta^{-4 H}\right| \leq C n^{4 H-3},
$$

where and in what follows $C>0$ denotes a generic constant independent of $n$ (but it may depend on $\theta, H$ ).

Proof From the definition of $F_{n}$ we see

$$
\begin{aligned}
\mathbb{E}\left(F_{n}^{2}\right) & =\frac{1}{n}\left[\sum_{k, k^{\prime}=1}^{n} \mathbb{E}\left(X_{k h}^{2} X_{k^{\prime} h}^{2}\right)-\sum_{k, k^{\prime}=1}^{n} \mathbb{E}\left(X_{k h}^{2}\right) \mathbb{E}\left(X_{k^{\prime} h}^{2}\right)\right] \\
& =\frac{2}{n} \sum_{k, k^{\prime}=1}^{n}\left[\mathbb{E}\left(X_{k h} X_{k^{\prime} h}\right)\right]^{2} \\
& =\frac{2}{n} \sum_{k \neq k^{\prime} ; k, k^{\prime}=1}^{n}\left[\mathbb{E}\left(X_{k h} X_{k^{\prime} h}\right)\right]^{2}+\frac{2}{n} \sum_{k=1}^{n}\left[\mathbb{E}\left(X_{k h}^{2}\right)\right]^{2} \\
& =A_{n}+B_{n} .
\end{aligned}
$$

We shall prove that $\lim _{n \rightarrow \infty} A_{n}=0$ and $\lim _{n \rightarrow \infty} B_{n}=2 \rho^{2} \theta^{-4 H}$. By Lemma 5.4 in [9], we have

$$
\begin{aligned}
A_{n} & \leq C \frac{1}{n} \sum_{k \neq k^{\prime}, k, k^{\prime}=1}^{n}\left|k-k^{\prime}\right|^{4 H-4} \\
& \leq C \frac{1}{n} \sum_{i=1}^{n} \sum_{j=i+1}^{n}(j-i)^{4 H-4} \\
& \leq C \frac{1}{n} \sum_{i=1}^{n}(n-i)^{4 H-3} \\
& \leq C n^{4 H-3}
\end{aligned}
$$


which implies that $\lim _{n \rightarrow \infty} A_{n}=0$ when $H<\frac{3}{4}$. On the other hand,

$$
\lim _{n \rightarrow \infty} B_{n}=2 \lim _{n \rightarrow \infty}\left(\mathbb{E} X_{n h}^{2}\right)^{2}=2 H^{2} \Gamma^{2}(2 H) \sigma^{4} \theta^{-4 H}=2 \rho^{2} \theta^{-4 H} .
$$

To prove the second inequality, it suffices to show that

$$
\left|\frac{1}{n} \sum_{k=1}^{n}\left[\mathbb{E}\left(X_{k h}^{2}\right)\right]^{2}-\rho^{2} \theta^{-4 H}\right| \leq C n^{4 H-3} .
$$

In fact,

$$
\begin{aligned}
& \left|\frac{1}{n} \sum_{k=1}^{n}\left[\mathbb{E}\left(X_{k h}^{2}\right)\right]^{2}-\rho^{2} \theta^{-4 H}\right| \\
\leq & \frac{1}{n} \sum_{k=1}^{n}\left|\mathbb{E}\left(X_{k h}^{2}\right)-\rho \theta^{-2 H}\right|\left(\mathbb{E}\left(X_{k h}^{2}\right)+\rho \theta^{-2 H}\right) \\
\leq & C \frac{1}{n} \sum_{k=1}^{n}\left|\mathbb{E}\left(X_{k h}^{2}\right)-\rho \theta^{-2 H}\right| .
\end{aligned}
$$

However, we have

$$
\begin{aligned}
& \left|\mathbb{E}\left(X_{k h}^{2}\right)-\rho \theta^{-2 H}\right| \\
= & C\left(\int_{0}^{\infty} \int_{0}^{s} e^{-\theta(u+s)}|s-u|^{2 H-2} d u d s-\int_{0}^{k h} \int_{0}^{s} e^{-\theta(u+s)}|s-u|^{2 H-2} d u d s\right) \\
= & C \int_{k h}^{\infty} \int_{0}^{s} e^{-\theta(u+s)}|s-u|^{2 H-2} d u d s \\
= & C \int_{k h}^{\infty} \int_{0}^{s} e^{\theta(x-2 s)} x^{2 H-2} d x d s \\
\leq & C \int_{k h}^{\infty} e^{\theta(-s)} s^{2 H-1} d s \\
\leq & C \int_{k h}^{\infty} e^{\theta(-s / 2)} d s \\
\leq & C e^{-k h / 2} .
\end{aligned}
$$

Hence, we have

$$
\left|\frac{1}{n} \sum_{k=1}^{n}\left[\mathbb{E}\left(X_{k h}^{2}\right)\right]^{2}-\rho^{2} \theta^{-4 H}\right| \leq C n^{-1} \leq C n^{4 H-3}
$$

which completes the proof.

Now we have

$$
D F_{n}=\frac{2}{\sqrt{n}} \sum_{k=1}^{n} X_{k h} D X_{k h} .
$$


Thus

$$
G_{n}:=\left\langle D F_{n}, D F_{n}\right\rangle_{\mathcal{H}}=\frac{4}{n} \sum_{k, k^{\prime}=1}^{n} X_{k h} X_{k^{\prime} h}\left\langle D X_{k h}, D X_{k^{\prime} h}\right\rangle_{\mathcal{H}} .
$$

Since $X_{k h}$ is normal random variable, it is easy to see that

$$
\left\langle D X_{k h}, D X_{k^{\prime} h}\right\rangle_{\mathcal{H}}=\mathbb{E}\left(X_{k h} X_{k^{\prime} h}\right) .
$$

Thus

$$
G_{n}=\frac{4}{n} \sum_{k, k^{\prime}=1}^{n} X_{k h} X_{k^{\prime} h} \mathbb{E}\left(X_{k h} X_{k^{\prime} h}\right)
$$

It is easy to check

$$
\mathbb{E}\left(G_{n}\right)=2 \mathbb{E}\left(F_{n}^{2}\right)
$$

which converges to $4 \rho^{2} \theta^{-4 H}$ as $n \rightarrow \infty$ by Lemma 4 .1. Thus to verify (ii) of Proposition 2.1, it suffices to show that

$$
\lim _{n \rightarrow \infty} \mathbb{E}\left[G_{n}-\mathbb{E}\left(G_{n}\right)\right]^{2}=0 .
$$

However,

$\mathbb{E}\left[G_{n}-\mathbb{E}\left(G_{n}\right)\right]^{2}=\mathbb{E}\left(G_{n}^{2}\right)-\left[\mathbb{E}\left(G_{n}\right)\right]^{2}$

$$
\begin{aligned}
= & \frac{1}{n^{2}} \sum_{k, k^{\prime} ; j, j^{\prime}=1}^{n}\left\{\mathbb{E}\left[X_{k h} X_{k^{\prime} h} X_{j h} X_{j^{\prime} h}\right] \mathbb{E}\left[X_{k h} X_{k^{\prime} h}\right] \mathbb{E}\left[X_{j h} X_{j^{\prime} h}\right]\right. \\
& \left.-\left(\mathbb{E}\left[X_{k h} X_{k^{\prime} h}\right] \mathbb{E}\left[X_{j h} X_{j^{\prime} h}\right]\right)^{2}\right\} .
\end{aligned}
$$

The expectation $\mathbb{E}\left(X_{1} X_{2} \cdots X_{p}\right)$ can be computed by the well-known Feynman diagram. In the case $p=4$, we have

$\mathbb{E}\left(X_{1} X_{2} X_{3} X_{4}\right)=\mathbb{E}\left(X_{1} X_{2}\right) \mathbb{E}\left(X_{3} X_{4}\right)+\mathbb{E}\left(X_{1} X_{3}\right) \mathbb{E}\left(X_{2} X_{4}\right)+\mathbb{E}\left(X_{1} X_{4}\right) \mathbb{E}\left(X_{2} X_{3}\right)$.

Thus

$\mathbb{E}\left[G_{n}-\mathbb{E}\left(G_{n}\right)\right]^{2}=\frac{32}{n^{2}} \sum_{k, k^{\prime}, j, j^{\prime}=1}^{n} \mathbb{E}\left[X_{k h} X_{j h}\right] \mathbb{E}\left[X_{k^{\prime} h} X_{j^{\prime} h}\right] \mathbb{E}\left[X_{k h} X_{k^{\prime} h}\right] \mathbb{E}\left[X_{j h} X_{j^{\prime} h}\right]$.

From Lemma 5.4 (Equation (5.7)) of [9], we have

$$
\left|\mathbb{E}\left[X_{k h} X_{k^{\prime} h}\right]\right| \leq \sigma^{2} C_{\theta, h, H}\left|k-k^{\prime}\right|^{2 H-2} .
$$


Therefore,

$$
\begin{aligned}
& \mathbb{E}\left[G_{n}-\mathbb{E}\left(G_{n}\right)\right]^{2} \\
\leq & \frac{C}{n^{2}} \sum_{k, k^{\prime}, j, j^{\prime}=1}^{n}|k-j|^{2 H-2}\left|k^{\prime}-j^{\prime}\right|^{2 H-2}\left|k-k^{\prime}\right|^{2 H-2}\left|j-j^{\prime}\right|^{2 H-2} \\
\leq & \frac{C}{n^{2}} \int_{[0, n]^{4}}|u-v|^{2 H-2}\left|u^{\prime}-v^{\prime}\right|^{2 H-2}\left|u-v^{\prime}\right|^{2 H-2}\left|v-v^{\prime}\right|^{2 H-2} d u d v d u^{\prime} d v^{\prime} \\
= & C n^{4(2 H-2)+4-2} \int_{[0,1]^{4}}|u-v|^{2 H-2}\left|u^{\prime}-v^{\prime}\right|^{2 H-2}\left|u-v^{\prime}\right|^{2 H-2}\left|v-v^{\prime}\right|^{2 H-2} d u d v d u^{\prime} d v^{\prime} \\
\leq & C n^{8 H-6}
\end{aligned}
$$

which converges to 0 as $n \rightarrow \infty$ if $H<3 / 4$.

Summarizing the above, we can state

Theorem 4.2 Let $X_{t}$ be the Ornstein-Uhlenbeck process defined by (1.2) and let $\xi_{n}$ be defined by (4.2). If $1 / 2 \leq H<3 / 4$, then

$$
\sqrt{n}\left(\xi_{n}-\mathbb{E}\left(\xi_{n}\right)\right) \rightarrow N(0, \Sigma),
$$

where

$$
\Sigma=\lim _{n \rightarrow \infty} \mathbb{E}\left(F_{n}^{2}\right)=2 \rho^{2} \theta^{-4 H} .
$$

To study the weak convergence of $\sqrt{n}\left(\hat{\theta}_{n}-\theta\right)$, we need the following lemma.

Lemma 4.3 Let $H \geq 1 / 2$. Then

$$
\sqrt{n}\left|\mathbb{E}\left(\xi_{n}\right)-\rho \theta^{-2 H}\right| \leq C n^{-\frac{1}{2}},
$$

and hence

$$
\lim _{n \rightarrow \infty} \sqrt{n}\left(\mathbb{E}\left(\xi_{n}\right)-\rho \theta^{-2 H}\right)=0 .
$$

Proof From the definition of $\xi_{n}$, we have

$$
\begin{aligned}
& \sqrt{n}\left|\mathbb{E}\left(\xi_{n}\right)-\rho \theta^{-2 H}\right| \\
= & \frac{C}{\sqrt{n}} \sum_{k=1}^{n}\left|\int_{0}^{k h} \int_{0}^{k h} e^{-\theta(u+s)}\right| u-\left.s\right|^{2 H-2} d s d u-\int_{0}^{\infty} \int_{0}^{\infty} e^{-\theta(u+s)}|u-s|^{2 H-2} d s d u \mid \\
= & \frac{C}{\sqrt{n}} \sum_{k=1}^{n}\left|\int_{0}^{k h} \int_{0}^{u} e^{-(u+s)}\right| u-\left.s\right|^{2 H-2} d s d u-\int_{0}^{\infty} \int_{0}^{u} e^{-(u+s)}|u-s|^{2 H-2} d s d u \mid \\
= & \frac{C}{\sqrt{n}} \sum_{k=1}^{n} \int_{\theta k h}^{\infty} \int_{0}^{u} e^{-(u+s)}|u-s|^{2 H-2} d s d u \\
= & \frac{C}{\sqrt{n}} \sum_{k=1}^{n} \int_{\theta k h}^{\infty} \int_{0}^{u} e^{-2 u+x} x^{2 H-2} d x d u \leq \frac{C}{\sqrt{n}} \sum_{k=1}^{n} \int_{\theta k h}^{\infty} e^{-2 u} e^{u} u^{2 H-1} d u \\
\leq & \frac{C}{\sqrt{n}} \sum_{k=1}^{n} \int_{\theta k h}^{\infty} e^{-\frac{1}{2} u} d u \leq \frac{C}{\sqrt{n}} \sum_{k=1}^{n} e^{-\frac{\theta k h}{2}} \leq \frac{C}{\sqrt{n}} .
\end{aligned}
$$


This proves the lemma.

Let us recall that

$$
\hat{\theta}_{n}=\left(\frac{\xi_{n}}{\rho}\right)^{-1 /(2 H)}
$$

Therefore

$$
\sqrt{n}\left(\hat{\theta}_{n}-\theta\right)=-\frac{1}{2 H} \tilde{\xi}_{n}^{-1 /(2 H)-1} \sqrt{n}\left(\frac{\xi_{n}}{\rho}-\theta^{-2 H}\right),
$$

where $\tilde{\xi}_{n}$ is between $\theta^{-2 H}$ and $\frac{\xi_{n}}{\rho}$. Since $\tilde{\xi}_{n} \rightarrow \theta^{-2 H}$ almost surely and since $\sqrt{n}\left(\xi_{n}-\rho \theta^{-2 H}\right)$ converges to $N(0, \Sigma)$ in law by Theorem 4.2 and Lemma 4.3, we see that $\sqrt{n}\left(\hat{\theta}_{n}-\theta\right)$ converges in law to

$$
N\left(0, \frac{\theta^{4 H+2}}{4 H^{2} \rho^{2}} \Sigma\right)=N\left(0, \frac{\theta^{2}}{2 H^{2}}\right) .
$$

Thus we arrive at our main theorem of this section.

Theorem 4.4 Let $1 / 2 \leq H<3 / 4$. Then

$$
\sqrt{n}\left(\hat{\theta}_{n}-\theta\right) \rightarrow N\left(0, \frac{\theta^{2}}{2 H^{2}}\right) \quad \text { in law } \quad \text { as } \quad n \rightarrow \infty .
$$

\section{Berry-Esseen asymptotics}

Theorem 4.4 shows that when $n \rightarrow \infty, Q_{n}:=\sqrt{\frac{2 H^{2} n}{\theta^{2}}}\left(\hat{\theta}_{n}-\theta\right)$ converges to $N(0,1)$ in law. In this section we shall obtain a rate of this convergence. We shall use Proposition 2.3. To this end we need to compute the 4-th cumulant $\kappa_{4}\left(F_{n}\right)$.

Let us develop a general approach to estimate $\kappa_{4}\left(Q_{n}\right)$ which is particularly useful for our situation. To simplify notation we omit the explicit dependence on $n$. It is clear that if $Z_{k}=\int_{0}^{T} f_{k}(s) d B_{s}^{H}$ for some (deterministic) $f_{k} \in \mathcal{H}$, then

$$
V=\sum_{k=1}^{N}\left(Z_{k}^{2}-\mathbb{E}\left(Z_{k}^{2}\right)\right)=\sum_{k=1}^{N} I_{2}\left(f_{k}^{\otimes 2}\right) .
$$

Thus

$$
f=\sum_{k=1}^{N} f_{k} \otimes f_{k}
$$

and

$$
H_{f}^{4}=\sum_{k_{1}, k_{2}, k_{3}, k_{4}=1}^{N} f_{k_{1}} \otimes f_{k_{4}}\left\langle f_{k_{1}}, f_{k_{2}}\right\rangle_{\mathcal{H}}\left\langle f_{k_{2}}, f_{k_{3}}\right\rangle_{\mathcal{H}}\left\langle f_{k_{3}}, f_{k_{4}}\right\rangle_{\mathcal{H}}
$$


which is a map from $\mathcal{H}$ to $\mathcal{H}$ such that for $g \in \mathcal{H}$,

$$
H_{f}^{4}(g)(t)=\sum_{k_{1}, k_{2}, k_{3}, k_{4}=1}^{N}\left\langle f_{k_{1}}, f_{k_{2}}\right\rangle_{\mathcal{H}}\left\langle f_{k_{2}}, f_{k_{3}}\right\rangle_{\mathcal{H}}\left\langle f_{k_{3}}, f_{k_{4}}\right\rangle_{\mathcal{H}}\left\langle f_{k_{4}}, g\right\rangle_{\mathcal{H}} f_{k_{1}}(t) .
$$

If $V$ is given by (5.1), then the 4 -th cumulant of $V$ is

$$
\begin{aligned}
\kappa_{4}(V) & =\operatorname{Tr}\left(H_{f}^{4}\right) \\
& =\sum_{k_{1}, k_{2}, k_{3}, k_{4}=1}^{N}\left\langle f_{k_{1}}, f_{k_{2}}\right\rangle_{\mathcal{H}}\left\langle f_{k_{2}}, f_{k_{3}}\right\rangle_{\mathcal{H}}\left\langle f_{k_{3}}, f_{k_{4}}\right\rangle_{\mathcal{H}}\left\langle f_{k_{4}}, f_{k_{1}}\right\rangle_{\mathcal{H}} \\
& =\sum_{k_{1}, k_{2}, k_{3}, k_{4}=1}^{N} \mathbb{E}\left(Z_{k_{1}} Z_{k_{2}}\right) \mathbb{E}\left(Z_{k_{2}} Z_{k_{3}}\right) \mathbb{E}\left(Z_{k_{3}} Z_{k_{4}}\right) \mathbb{E}\left(Z_{k_{4}} Z_{k_{1}}\right) .
\end{aligned}
$$

If we apply this computation (5.3) to $F_{n}$ defined in Section 4 , then we see that $\kappa_{4}\left(F_{n}\right)$ is the same as $\mathbb{E}\left(G_{n}-\mathbb{E}\left(G_{n}\right)\right)^{2}$ studied in Section 4. Thus we have from (4.6)

$$
\kappa_{4}\left(F_{n}\right) \leq C n^{8 H-6} .
$$

By Lemma 4.1, we have

$$
\left|\kappa_{2}\left(F_{n}\right)-\Sigma\right|=\left|E\left(F_{n}^{2}\right)-\Sigma\right| \leq C n^{4 H-3} .
$$

Therefore by Proposition 2.3, we have

\section{Lemma 5.1}

$$
\sup _{z \in \mathbb{R}}\left|P\left(-\frac{F_{n}}{\sqrt{\Sigma}} \leq z\right)-\Psi(z)\right| \leq C n^{4 H-3},
$$

where $\Psi(z)=\frac{1}{\sqrt{2 \pi}} \int_{-\infty}^{z} e^{-\frac{x^{2}}{2}} d x$ is the error function.

We also have the following lemma.

Lemma 5.2 Let $1 / 2 \leq H<3 / 4$. There is a constant $C$ such that

$$
\sup _{y \in \mathbb{R}}\left|P\left(\sqrt{\frac{n}{2}} \theta^{2 H}\left(\theta^{-2 H}-\hat{\theta}_{n}^{-2 H}\right) \leq y\right)-\Psi(y)\right| \leq C n^{(4 H-3) \vee\left(-\frac{1}{2}\right)} .
$$

Proof Recall that $F_{n}=\sqrt{n}\left(\xi_{n}-\mathbb{E}\left(\xi_{n}\right)\right)$.

Let $\tilde{F}_{n}=\sqrt{n}\left(\xi_{n}-\rho \theta^{-2 H}\right)$, and $a_{n}=\tilde{F}_{n}-F_{n}=\sqrt{n}\left(\mathbb{E}\left(\xi_{n}\right)-\rho \theta^{-2 H}\right)$, then $\left|a_{n}\right| \leq C n^{-\frac{1}{2}}$ by Lemma 4.3 .

$$
\begin{aligned}
& \left|P\left(-\frac{\tilde{F}_{n}}{\sqrt{\Sigma}} \leq z\right)-\Psi(z)\right| \\
= & \left|P\left(-\frac{F_{n}+a_{n}}{\sqrt{\Sigma}} \leq z\right)-\Psi(z)\right| \\
\leq & \left|P\left(-\frac{F_{n}}{\sqrt{\Sigma}} \leq z+\frac{a_{n}}{\sqrt{\Sigma}}\right)-\Psi\left(z+\frac{a_{n}}{\sqrt{\Sigma}}\right)\right|+\left|\Psi\left(z+\frac{a_{n}}{\sqrt{\Sigma}}\right)-\Psi(z)\right| \\
\leq & C\left(n^{4 H-3}+n^{-\frac{1}{2}}\right) .
\end{aligned}
$$


The inequality (5.4) is obtained since $\xi_{n}=\rho \hat{\theta}_{n}^{-2 H}$ and $\Sigma=2 \rho^{2} \theta^{-4 H}$.

Now we can prove our main theorem.

Theorem 5.3 Let $1 / 2 \leq H<3 / 4$. For any $K>0$, there exist a constant $C_{K}$ depending on $K$ and $H$, and a constant $N_{K}>0$ depending on $K$, such that when $n>N_{K}$,

$$
\sup _{|z| \leq K}\left|P\left(\frac{\sqrt{2 n} H}{\theta}\left(\hat{\theta}_{n}-\theta\right) \leq z\right)-\Psi(z)\right| \leq C_{K} n^{(4 H-3) \vee\left(-\frac{1}{2}\right)} .
$$

Proof Now we have

$$
\begin{aligned}
& P\left(\sqrt{\frac{n}{2}} \theta^{2 H}\left(\theta^{-2 H}-\hat{\theta}_{n}^{-2 H}\right) \leq y\right) \\
= & P\left(\hat{\theta}_{n} \leq \theta\left(1-\sqrt{\frac{2}{n}} y\right)^{-\frac{1}{2 H}}\right) \\
= & P\left(\frac{\sqrt{2 n} H}{\theta}\left(\hat{\theta}_{n}-\theta\right) \leq \sqrt{2 n} H\left[\left(1-\sqrt{\frac{2}{n}} y\right)^{-\frac{1}{2 H}}-1\right]\right) .
\end{aligned}
$$

Choose $y_{n, z}$ so that

$$
\sqrt{2 n} H\left[\left(1-\sqrt{\frac{2}{n}} y_{n, z}\right)^{-\frac{1}{2 H}}-1\right]=z
$$

namely,

$$
y_{n, z}=\sqrt{\frac{n}{2}}\left[1-\left(1+\frac{z}{\sqrt{2 n} H}\right)^{-2 H}\right] .
$$

Then

$$
\begin{aligned}
& \left|P\left(\frac{\sqrt{2 n} H}{\theta}\left(\hat{\theta}_{n}-\theta\right) \leq z\right)-\Psi(z)\right| \\
= & \left|P\left(\sqrt{\frac{n}{2}} \theta^{2 H}\left(\theta^{-2 H}-\hat{\theta}_{n}^{-2 H}\right) \leq y_{n, z}\right)-\Psi(z)\right| \\
\leq & \left|P\left(\sqrt{\frac{n}{2}} \theta^{2 H}\left(\theta^{-2 H}-\hat{\theta}_{n}^{-2 H}\right) \leq y_{n, z}\right)-\Psi\left(y_{n, z}\right)\right|+\left|\Psi\left(y_{n, z}\right)-\Psi(z)\right| .
\end{aligned}
$$

The inequality (5.4) implies that the above first term is bounded by $C n^{(4 H-3) \vee\left(-\frac{1}{2}\right)}$. It is easy to check that there exits a constant $C_{K}$ depending on $K$ and $H$, and a number $N_{K}$ depending on $K$, such that when $n>N_{K},\left|\Psi\left(y_{n, z}\right)-\Psi(z)\right| \leq$ $\left|y_{n, z}-z\right| \leq C_{K} n^{-1 / 2}$ for all $|z| \leq K$. 
Remark 5.4 Throughout this paper we did not discuss the case $H=1 / 2$ in detail, which is easy.

Acknowledgment We appreciate Chihoon Lee and the referee's careful reading of this paper.

\section{References}

[1] Biagini, F.; Hu, Y.; Øksendal, B. and Zhang, T. Stochastic calculus for fractional Brownian motion and applications. Springer, 2008.

[2] Cheridito, P.; Kawaguchi H. and Maejima, M. Fractional OrnsteinUhlenbeck processes. Electronic Journal of Probability 8 (2003) 1-14.

[3] Dacunha-Castelle, D. and Florens-Zmirou, D. Estimation of the coefficients of a diffusion from discrete observations. Stochastics 19 (1986), no. 4, 263284.

[4] Duncan, T. E.; Hu, Y. and Pasik-Duncan, B. Stochastic calculus for fractional Brownian motion. I. Theory. SIAM J. Control Optim. 38 (2000) $582-612$.

[5] Fox, R. and Taqqu, M. S. Central limit theorems for quadratic forms in random variables having long-range dependence. Probab. Theory Related Fields 74 (1987), 213-240.

[6] $\mathrm{Hu}, \mathrm{Y}$. Integral transformations and anticipative calculus for fractional Brownian motions. Mem. Amer. Math. Soc. 175 (2005), 825.

[7] Hu, Y. and Long, H. Least squares estimator for Ornstein-Uhlenbeck processes driven by $\alpha$-stable motions. Stochastic Process. Appl. 119 (2009), 2465-2480.

[8] Hu, Y. and Long, H. Least squares estimator for O rnstein-Uhlenbeck processes driven by $\alpha$-stable motions. Stochastic Process. Appl. 119 (2009), no. $8,2465-2480$.

[9] Hu, Y. and Nualart, D. Parameter estimation for fractional OrnsteinUhlenbeck processes. Statist. Probab. Lett. 80 (2010), no. 11-12, 1030-1038.

[10] Liptser, R.S. and Shiryaev, A.N. Statistics of Random Processes: II Applications. Second Edition, Applications of Mathematics, Springer, 2001.

[11] Neveu, J. Processus aléatoires gaussiens. Les Presses de l'Universit de Montral, Montreal, Que. 1968.

[12] Nourdin, I. and Peccati, G. Stein's method and exact Berry-Esseen asymptotics for functionals of Gaussian fields. Ann. Probab. 37 (2009), 2231-2261. 
[13] Nualart, D. The Malliavin Calculus and Related Topics. Second edition, Springer, 2006.

[14] Nualart, D. and Ortiz-Latorre, S. Central limit theorems for multiple stochastic integrals and Malliavin calculus. Stochastic Process. Appl. 118 (2008) 614-628.

[15] Prakasa Rao, B. L. S. Statistical inference for diffusion type processes. Kendall's Library of Statistics, 8. Oxford University Press, 1999.

[16] Skorohod A. V. On a generalization of a stochastic integral. Theory Probab. Appl. 20 (1975), 219-233.

Yaozhong $\mathrm{Hu}$

Department of Mathematics

University of Kansas

Lawrence, Kansas, 66045

hu@math.ku.edu

and

Jian Song

Department of Mathematics

Rutgers University

Piscataway, NJ 08854-8019

jsong2@math.rutgers.edu 